\title{
Review of: "Comparison of nasal patency after nose- blowing between pinch versus no pinch method: a randomized controlled trial"
}

\author{
Zheng Zhu
}

Potential competing interests: The author(s) declared that no potential competing interests exist.

To the Editor:

Patorn Piromchai et al (1), in their article recently published in Scientific Reports, compared nasal patency after nose-blowing between pinch and no pinch method, the data showed that there was no difference in nasal patency evaluated by using acoustic rhinometry while the patients felt that their nose was clearer when pinching and blowing. We would like to comment. Nasal obstruction, stream, sneeze and itch are typical symptoms of allergic rhinitis patients and subjects with cold. The above symptoms of disease severely impaired quality of life of the patients, especially with nasal obstruction and stream as the patients need frequent nose-blowing during daily work or social activation (2). Different patients may have different symptoms, mainly with one or two of above symptoms (3). There are objective and subjective methods for evaluating the severity of these symptoms, especially nasal patency; Nasal airway resistance, acoustic rhinometry and peak nasal inspiratory flow are objective methods that mainly for the evaluation of nasal patency or obstruction, and symptom scores measured by visual analogue scale (VAS) is subjective method that for the evaluation of stream, sneeze and itch $(4,5)$. The combination use of objective and subjective methods can well evaluate patients with different symptoms $(3,6)$. Nasal mucosal or secretion may contribute to nasal obstruction. We should evaluate obstruction and stream scores separately both before and after nasal-blowing to evaluate how much nasal mucosal secretion contribute to nasal obstruction. If the patient was mainly with obstruction (mucosal swelling) but without much secretion, the pinch and blow may have little effect on reducing nasal obstruction. We may measure the volume or weight of nasal discharge for evaluate the efficacy of nasal-blowing on reducing nasal patency. We think nasal airway resistance rather than cross-sectional area and length of the nose may directly reflect and well correlate with nasal patency in allergic rhinitis patients or cold. And when measure nasal airway resistance, we should measure the resistance of each side of nasal and calculate total nasal airway resistance, which may exclude the impact of nasal cycle. The above may partially explain why there was no difference in nasal patency evaluated by using objective method (acoustic rhinometry) while the patients felt (VAS) that their nose was clearer when pinching and blowing.

\section{References}

1. Piromchai P, Netnoi J, Srirompotong S, Thanawirattananit P. Comparison of nasal patency after nose- 
blowing between pinch versus no pinch method: a randomized controlled trial. Sci Rep. 2021;11(1):22084.

2. Bousquet J, Khaltaev N, Cruz AA, Denburg J, Fokkens WJ, Togias A et al. Allergic Rhinitis and its Impact on Asthma (ARIA) 2008 update (in collaboration with the World Health Organization, GA(2)LEN and AllerGen). Allergy 2008;63(Suppl 86):8-160.

3. Riechelmann H, Bachert C, Goldschmidt O, Hauswald B, Klimek L, Schlenter WW et al. Application of the nasal provocation test on diseases of the upper airways. Position Paper of the German Society for Allergology and Clinical Immunology (ENT Section) in cooperation with the Working Team for Clinical Immunology. Laryngo-Rhino-Otol 2003;82:183-188.

4. Dor-Wojnarowska A, Radlińska A, Rabski M, Fal A, Liebhart J, Zaleska A et al. Investigation of Repeatability of Peak Nasal Inspiratory Flow Rate Measurements Under Baseline Conditions and After Administration of 0.05\% Oxymetazoline. Am J Rhinol Allergy. 2022;36(1):41-46.

5. Kim YH, Jang TY. Proposed diagnostic standard using visual analogue scale and acoustic rhinometry in nasal provocation test in allergic rhinitis. Auris Nasus Larynx 2011;38:340-346.

6. Zhu Z, Xie Y, Guan W, Gao Y, Xia S, Huang R et al. Effects of leukotriene D4 and histamine nasal challenge on airway responsiveness and inflammation in persistent allergic rhinitis patients. Clin Respir J. 2018;12(2):587-594

Zheng Zhu PhD, Shu Xia² PhD, Mo Xian ${ }^{1}$ PhD

1. Department of Allergy and Clinical Immunology, Guangzhou Institute of Respiratory Health, State Key Laboratory of Respiratory Disease, The First Affiliated Hospital of Guangzhou Medical University, Guangzhou, China

2. Department of Respiratory and Critical Care Medicine, Guangzhou Institute of Respiratory Health, State Key Laboratory of Respiratory Disease, The First Affiliated Hospital of Guangzhou Medical University, Guangzhou, China

All authors participated in drafting and reviewing of this manuscript. All authors read and approved the final version of the article. The authors have disclosed that they do not have any potential conflicts of interest. 